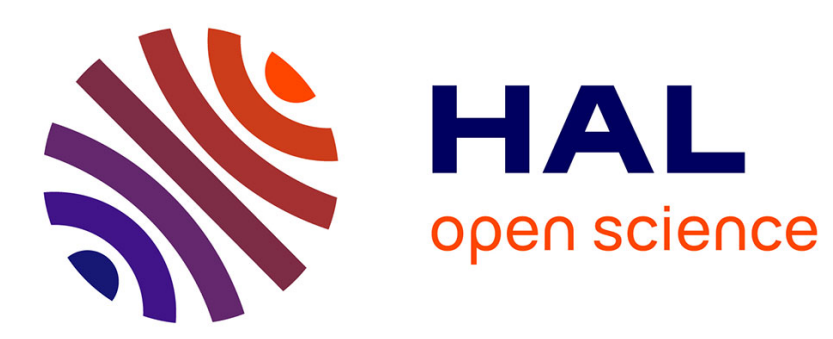

\title{
Scalable Data Hiding for Online Textured 3D Terrain Visualization
}

Khizar Hayat, William Puech, Gilles Gesquière

\section{To cite this version:}

Khizar Hayat, William Puech, Gilles Gesquière. Scalable Data Hiding for Online Textured 3D Terrain Visualization. ICME: International Conference on Multimedia and Expo, Jun 2008, Hannover, Germany. pp.217-220. lirmm-00293106

\section{HAL Id: lirmm-00293106 https://hal-lirmm.ccsd.cnrs.fr/lirmm-00293106}

Submitted on 3 Jul 2008

HAL is a multi-disciplinary open access archive for the deposit and dissemination of scientific research documents, whether they are published or not. The documents may come from teaching and research institutions in France or abroad, or from public or private research centers.
L'archive ouverte pluridisciplinaire HAL, est destinée au dépôt et à la diffusion de documents scientifiques de niveau recherche, publiés ou non, émanant des établissements d'enseignement et de recherche français ou étrangers, des laboratoires publics ou privés. 


\section{SCALABLE DATA HIDING FOR ONLINE TEXTURED 3D TERRAIN VISUALIZATION}

\author{
Khizar Hayat, William Puech \\ LIRMM, UMR CNRS 5506 \\ University of Montpellier II \\ 34392 MONTPELLIER FRANCE
}

Gilles Gesquière

\author{
LSIS, UMR CNRS 6168 \\ Aix-Marseille University \\ 13200 ARLES FRANCE
}

\begin{abstract}
A method for 3D scalable visualization, in a client/server environment is presented. The main idea presented in this paper is to increase the quality of 3D visualization for low bit rate transmission. All informations like texture, digital elevation model and projection systems are merged into a single file. The integration is achieved via data hiding whereas the scalability is realized through the multiresolution nature of JPEG2000 encoding. The embedding step is done in the lossless DWT domain. The strategy is flexible and it is up to the user to decide the level of transform of texture and DEM. In this context a comparison between various possibilities is presented by applying the method to a practical example. It is shown that a very good visualization can be realized with even a tiny fraction of the encoded coefficients.
\end{abstract}

Index Terms - 3D visualization, scalable data-hiding, Discrete Wavelet Transform (DWT), JPEG2000, digital elevation model (DEM), Geographic Information System (GIS), Data synchronization.

\section{INTRODUCTION}

Terrain visualization in three dimensions requires at least two file: texture images in some form like aerial photograph and a set of coefficients obtained by digitizing the geometry of terrain, called digital elevation model (DEM). Each of the DEM coefficient represents the altitude of a particular square block of texture pixels and the process of visualization is the overlaying of texture over a regular triangle network $[1,2]$ obtained from the DEM. With today technology, the 3D visualization quality may be very high but the client/server environments are very diverse in terms of network, computation and memory resources. For catering each of the perspective client, it is advisable to encode the data in a scalable way, unified into one standard format file. The JPEG2000 format [3] offers the scalability thanks to the multiresolution nature of its discrete wavelet transform (DWT). For the integration of all the data into one file one can rely on the technique of data hiding due to the smaller size of the DEM file as it can be embedded in the bulky texture image. But this embedding must be carried out in such a way that the JPEG2000 file format is conserved and there is no need of any new format. In addition the embedding must not interfere with the scalability and for each of the possible resolutions, the corresponding texture and its DEM must be recaptured at the decoder.

Many methods have been proposed in the literature for wavelet-based data hiding but few of these are compatible with the JPEG2000 scheme. According to [4], data hiding methods for JPEG2000 images must process the code blocks independently. The blind scheme proposed in [5] is to integrate data hiding with the "Embedded Block Coding with Optimized Truncation (EBCOT)" and embed data during the formation of compressed bit stream. The scheme is claimed to have robustness and good perceptual transparency. One particular technique [6] embed watermark in the JPEG2000 pipeline after the stages of quantization and region of interest (ROI) scaling but before the entropy coding. Piva et al. have proposed an authentication scheme that embeds an image digest in a subset of the subbands from the DWT domain. The image digest is derived from the discrete cosine transformation (DCT) of the level 1 DWT $L L$ subband of the image after some processing [7]. One blind method [8] transforms the original image by one-level wavelet transform and sets the three higher subbands to zero before inverse transforming it to get the reference image. The difference values are used to ascertain the potential embedding locations of which a subset is selected randomly for embedding. The method of Kong et al. [9] embeds watermark in the weighted mean of the wavelets blocks, rather than in the individual coefficient, to make it robust and perceptually transparent. Of the various practical efforts, to integrate the visualization data, are solutions like GeoJP2 [10] and GMLJP2 [11] but these serve the purpose partially since the data is not synchronized and there is an increase in the original size of the JPEG2000 file. We follow a different course, to have the advantage of synchronization without any change in the JPEG2000 file size, by applying a scalable data hiding algorithm.

The rest of the paper is arranged as follows. Our proposed method is explained in Section 2. We present and analyze results in Section 3 and Section 4 concludes this paper. 


\section{GENERALIZED METHOD FOR SCALABLE HIDING OF 3D DATA}

This work is a continuation of our previous efforts [12] for the synchronized unification of the DEM and texture wherein DWT decomposition level of both the message (transformed DEM) and the carrier (transformed Y plane of the texture) were the same before embedding. Because of the sensitive nature of DEM it will be expedient to explore other ways to avoid an important decrease of its quality. The significant difference in the sizes of DEM and texture allows us the luxury to use much smaller texture blocks for embedding. The main idea presented in this paper is to increase the quality of the reconstructed DEM for a low bit rate transmission. At present block size of the texture is much higher than the size of a single DEM coefficient in bits. Since the number of required blocks is fixed, size reduction will automatically exclude some higher subbands from the embedding process. The strategy has to be then to use a subset rather than all the texture subbands of wavelet decomposition for embedding. The level of DWT decomposition of the DEM would then obviously be lesser than that of the texture. Since the packet order in a JPEG2000 stream is from low to high subbands, one is compelled to prefer the lowest subbands for embedding.

Keeping in view the above arguments, we propose, in this paper, a new method for a scalable transfer and on-line visualization of textured 3D terrain data. In the spatial domain, let one DEM coefficient corresponding to a $b \times b$ pixel block of the related texture. Suppose the texture is to be JPEG2000 coded at DWT decomposition level $L$, implying $R=L+1$ resolutions. Let us treat the set of DEM coefficients as a gray-scale image and apply lossless DWT at level $L^{\prime}$, where $L^{\prime} \leq L$. For embedding we interrupt the JPEG2000 coding after the DWT step, as illustrated Fig. 1, and get the transformed $Y C_{r} C_{b}$ components for embedding. If $L^{\prime}=L$ then block size is unchanged for embedding since all the subbands are included for possible data insertion. Otherwise, for

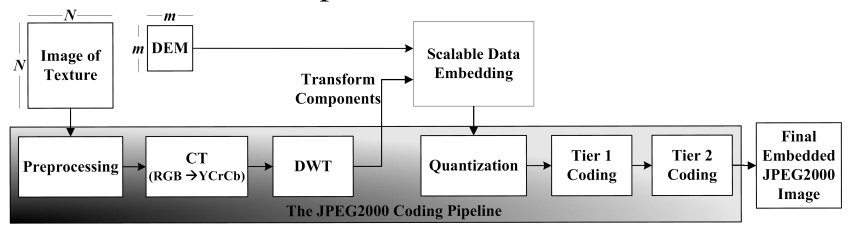

Fig. 1: Overview of the proposed method

$L^{\prime}<L$, only a subset subbands are used for embedding and the block size has to be reduced to $b / 2^{\left(L-L^{\prime}\right)} \times b / 2^{\left(L-L^{\prime}\right)}$. In fact only the lower $3 L^{\prime}+1$ of the original $3 L+1$ are used and the higher $3\left(L-L^{\prime}\right)$ unused. Care must be taken of the fact that block size must be large enough to have at least as many coefficients as are the number of bits used to represent a single DEM coefficient. Otherwise one would have to embed more than one bit per coefficient in a given block and/or even include the $C_{r}$ and $C_{b}$ planes for embedding. The process of embedding is elaborated in Algorithm 1 wherein one coefficient is embedded per $b / 2^{\left(L-L^{\prime}\right)} \times b / 2^{\left(L-L^{\prime}\right)}$ block, using the one or more LSBs of the selected coefficient for embedding.

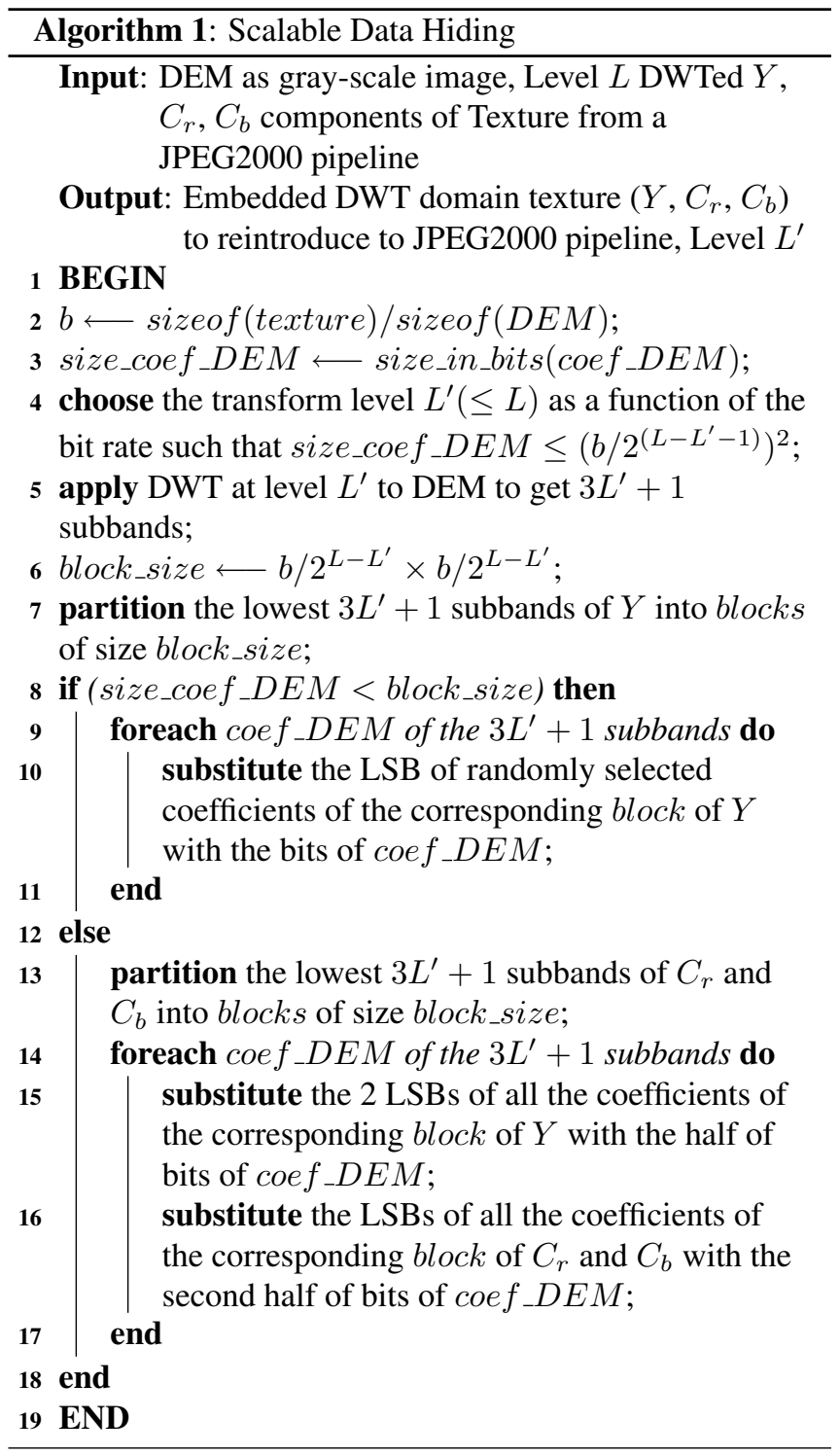

Two decisions are important during the embedding process. First is the level of DWT $\left(L^{\prime}\right)$ before embedding which is a trade off between the final texture quality and its DEM quality. At the decoding end the quality of the DEM would depend on the difference between $L$ and $L^{\prime}$. The larger the difference, $\left(L-L^{\prime}\right)$, higher will be the quality of the reconstructed DEM for a low bit rate transmission. The second decision concerns the allocation policy which answers issues like which component(s) out of the $Y C_{r} C_{b}$ should be used for embedding. We have also to analyze the maximum number of modifiable LSBs per coefficient and per component and what is the order if embedding amongst the allocated coefficients from the selected components. For the sake of readability, in Algorithm 1, the maximum number of modifiable LSBs of the same coefficient is restricted to 2 per $Y$ plane and 1 each of $C_{r}$ and $C_{b}$ planes. In other words we have set a limit of $1 / 4$ 
times the word-size of DEM coefficient on the block_size for embedding.

The above coded image can be utilized like any other JPEG2000 image and sent across any communication channel. The blind decoding is the reverse of the above process. Just before the inverse DWT stage of the JPEG2000 decoder the DEM can be blindly extracted using the above mentioned partitioning scheme. All the DEM bits are LSBs of the coefficients from the lowest $3 L^{\prime}+1$ subbands of one or more of the transformed texture components $Y C_{r} C_{b}$. One advantage of the method is in the fact that the DEM and texture can be reconstructed with even a small subset of the coefficients of the carrier. The resolution scalability of wavelets and the synchronized character of our method enable a $3 D$ visualization even with fewer than original resolution layers as a result of partial or delayed data transfer. The method thus enables to effect visualization from a fraction of data in the form of the lowest subband, of a particular resolution level. It is always possible to stuff 0's for the higher bands.

\section{RESULTS}

We have applied our method to a $2048 \times 2048$ pixel exam$\mathrm{ple}^{1}$ texture image (Fig. 2.b) and its corresponding DEM of $64 \times 64,16$ bit altitudes implying one altitude per $32 \times 32$ pixel texture block. The DEM is converted into a gray-scale image (Fig. 2.a) with whiter parts of the image representing the high altitudes, and black parts the low altitudes. A 3D visualization based on the two data is depicted in Fig. 2.c.

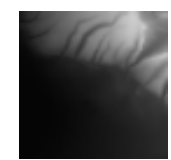

(a)

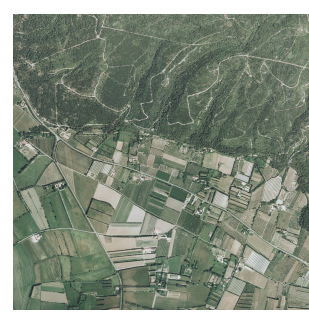

(b)

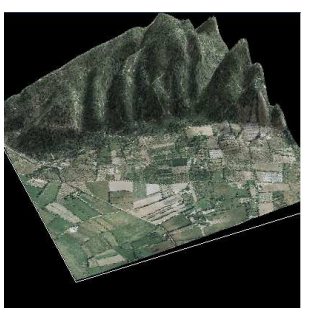

(c)
Fig. 2: Original data : a) DEM as an image, b) Texture image, c) $3 \mathrm{D}$ visualization with original data.

We chose to subject the texture to reversible JPEG2000 encoding at $L=5$ that would give us six possible resolutions. The condition $L^{\prime} \leq L$ implies that we can apply lossless DWT to the DEM image at five different levels before embedding. The embedding was done by interrupting the JPEG2000 coder just after DWT stage and getting the components which are $Y C_{r} C_{b}$ in this case. The $L^{\prime}$-transformed

\footnotetext{
${ }^{1}$ provided by IGN France (http://www.ign.fr/)
}

DEM was embedded in the lower $3 L^{\prime}+1$ subbands of one or more of the $Y C_{r} C_{b}$ components of the transformed texture in accordance with Algorithm 1. Upon the reintroduction of the altered components to the JPEG2000 pipeline we get our embedded texture image in JPEG2000 format. For $L^{\prime}>1$ the block size for embedding a 16 bit DWTed DEM coefficient is enough to allow for the substitution of at most one coefficient of the $3 L^{\prime}+1$ lower subbands of the most resilient $Y$ texture component. Hence there is no need to tamper with the $C_{r}$ or $C_{b}$ component. But for $L^{\prime}=1$ the block size is reduced to $2 \times 2$ in which 16 bits are to be embedded. The solution can be either to embed all in the 4 lowest subbands of the $Y$ component thus altering 4 LSBs of each coefficient (the $4 Y$ case $)^{2}$ or distribute in all the 3 components, e.g. 2 per $Y$ coefficient and 1 each per $C_{r}$ and $C_{b}$ in each of the coefficient of the lowest 4 subbands. A suitable key may be utilized to initialize a PRNG for deciding the order of embedding.

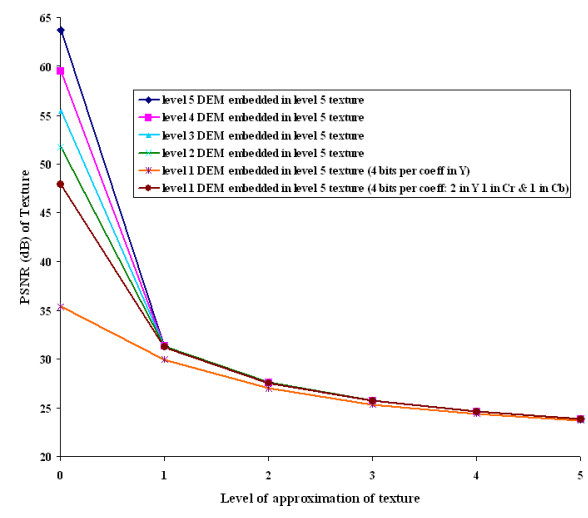

Fig. 3: Variation in texture quality as a function of the level of approximation of Texture.

The embedded texture on decoding can yield six different approximation images. A level $l(\leq L)$ approximation image is the one that is constructed with $\left(1 / 4^{l}\right) \times 100$ percent of the total coefficients that corresponds to the available lower $3 l+1$ subbands. For example, level 0 approximation image is constructed from all the coefficients and level 2 approximation image is constructed from $6.12 \%$ of the count of the initial coefficients. With our example we can have thirty possible approximation images. Fig. 3 shows the variation in the quality of the texture image as a function of the level of approximation for each of the five possible embedded textures. It must be noted that there are two main sources of quality loss. Firstly, the loss due to missing coefficients since barring level 0 for the rest of approximation images a fraction of transmitted coefficients are included in the decoding process and theses loss coefficients will obviously take away some quality. The second source of loss is the degradation of the lower subbands due to embedding. Since lower subbands have most of the image information, any tampering

\footnotetext{
${ }^{2}$ Note that the $4 Y$ case is mentioned here for the sake of comparison although it is not covered by Algorithm 1
} 
with their coefficients result in considerable quality loss. The first source has no role to play in case of level 0 approximation as all the coefficients are utilized in decoding. The curves in Fig. 3 and 4 show that quality loss due to missing coefficients is more marked than that due to embedding.

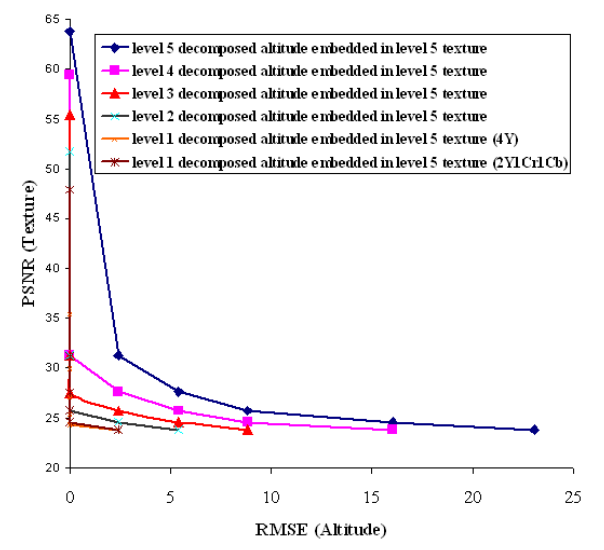

Fig. 4: Variation in texture quality as a function of the RMSE of DEM.

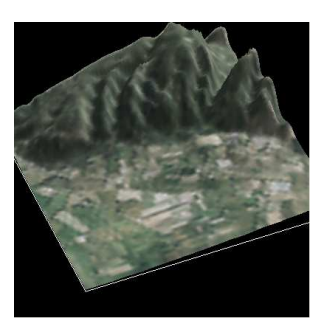

(a)

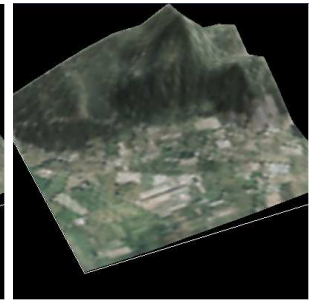

(b)

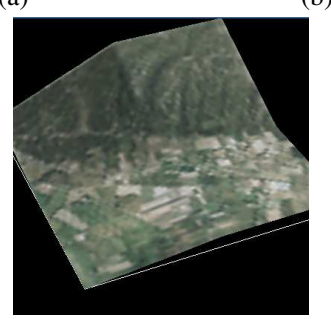

(c)

Fig. 5: 3D Visualization with a low bit rate transmission based on the level 5 approximation image when level $L^{\prime}$ DWTed DEM is embedded in level 5 DWTed texture: a) $L^{\prime}=1$, b) $L^{\prime}=3$, c) $L^{\prime}=5$.

To find a compromise between the competing texture and DEM quality, a plot of the PSNR of the reconstructed texture against the root mean square error (RMSE) in case of reconstructed DEM, as shown in Fig. 4, must be helpful. The Fig. 5 complete the previous graph with visual results.

\section{CONCLUSION}

The application of our method to a practical example illustrates the scalability in function of the network bit rate. For example, a client at desktop computer may have enough computing power and memory at his disposal to process all the transmitted coefficients and get the highest quality visualization. On the other hand an outdoor client with a smart-phone and $3 \mathrm{G}$ connection may have fewer resources to process all the data and has to content with a fraction of data but still can have optimal visualization. Besides our method allows for the integrity of the vital part of the information, i.e. DEM, although at the expense of a relatively insignificant loss in texture quality.

\section{ACKNOWLEDGMENT}

This work was supported in part by the Higher Education Commission (HEC) of Pakistan.

\section{REFERENCES}

[1] F. Losasso and H. Hoppe, "Geometry Clipmaps: Terrain Rendering Using Nested Regular Grids," ACM Trans. Graph., vol. 23, no. 3, pp. 769-776, 2004.

[2] R. Pajarola and E. Gobbetti, "Survey on Semi-Regular Multiresolution Models for Interactive Terrain Rendering," The Visual Computer, vol. 23, no. 8, pp. 583-605, 2007.

[3] ISO/IEC, ISO/IEC 15444-1: Information technology, JPEG2000 image coding system, Part 1: Core coding system, ISO Central Secretariat: CH-1211 Geneva, Switzerland, 2004.

[4] P. Meerwald and A. Uhl, "A Survey of Wavelet-domain Watermarking Algorithms," in Proc. of SPIE, Electronic Imaging, Security and Watermarking of Multimedia Contents III, San Jose, CA, USA, January 2001, vol. 4314, SPIE, IS\&T.

[5] P.-C. Su, H.-J. Wang, and C.-C. J. Kuo, "An Integrated Approach to Image Watermarking and JPEG-2000 Compression," Journal of VLSI Signal Processing Systems, vol. 27, no. 1-2, pp. 35-53, June 1997.

[6] P. Meerwald, "Quantization watermarking in the JPEG2000 coding pipeline," in Communications and Multimedia Security Issues of The New Century, IFIP TC6/TC11 Fifth Joint Working Conference on Communications and Multimedia Security, CMS '01, May 2001, pp. 69-79, Kluver Academic Publishing.

[7] A. Piva, F. Bartolini, and R. Caldelli, "Self Recovery Authentication of Images in The DWT Domain," Int. J. Image Graphics, vol. 5, no. 1, pp. 149-166, 2005.

[8] J.-L. Liu, D.-C. Lou, M.-C. Chang, and H.-K. Tso, "A Robust Watermarking Scheme Using Self-Reference Image," Computer Standards \& Interfaces, vol. 28, pp. 356-367, 2006.

[9] X. Kong, Y. Liu, H. Liu, and D. Yang, "Object Watermarks for Digital Images and Video," Image and Vision Computing, vol. 22, pp. 583-595, 2004.

[10] M. P. Gerlek, The "GeoTIFF Box" Specification for JPEG 2000 Metadata - DRAFT version 0.0, LizardTech, Inc. 1008 Western Ave Suite 200 Seattle, WA 98104 USA, April 2004.

[11] R. Lake, D. Burggraf, M. Kyle, and S. Forde, GML in JPEG 2000 for Geographic Imagery (GMLJP2) Implementation Specification, Number OGC 05-047r2. Open Geospatial Consortium (OGC), 2005.

[12] K. Hayat, W. Puech, and G. Gesquière, "An Efficient DataHiding Method Based on Lossless JPEG2000 for a Scalable and Synchronized Visualization of 3D Terrains," in 15th EUSIPCO'07, pp. 2519-2523, Poznan, Poland, sep. 2007. 